\title{
ILCEA
}

Revue de l'Institut des langues et cultures

d'Europe, Amérique, Afrique, Asie et Australie

19 | 2014

Acceptabilité et transgression en langues et cultures de spécialité

\section{Chartreuse verte, digestif du Moyen Âge devenu cocktail branché de la jeunesse française : un discours promotionnel séculaire face au cinéma américain}

Green Chartreuse, the Medicinal Liqueur from the Middle Ages Transformed into Today's Trendy Cocktail: A Centuries-Old Promotional Discourse Dethroned by the American Cinema

\section{Shaeda Isani}

\section{OpenEdition}

\section{Journals}

Édition électronique

URL : http://journals.openedition.org/ilcea/2399

DOI : 10.4000/ilcea.2399

ISSN : 2101-0609

Éditeur

UGA Éditions/Université Grenoble Alpes

Édition imprimée

ISBN : 978-2-84310-280-6

ISSN : 1639-6073

Référence électronique

Shaeda Isani, « Chartreuse verte, digestif du Moyen Âge devenu cocktail branché de la jeunesse

française : un discours promotionnel séculaire face au cinéma américain », ILCEA [En ligne], 19 | 2014, mis en ligne le 27 juin 2014, consulté le 01 mai 2019. URL : http://journals.openedition.org/ilcea/2399 ; DOI : 10.4000/ilcea.2399

Ce document a été généré automatiquement le 1 mai 2019.

(C) ILCEA 


\section{Chartreuse verte, digestif du} Moyen Âge devenu cocktail branché de la jeunesse française : un discours promotionnel séculaire face au cinéma américain

Green Chartreuse, the Medicinal Liqueur from the Middle Ages Transformed into Today's Trendy Cocktail: A Centuries-Old Promotional Discourse Dethroned by the American Cinema

Shaeda Isani

1 La présente recherche se situe dans le cadre d'une réflexion menée depuis 2006 sur les secteurs d'activité commerciale dits du "péché » ou du "vice », et notamment sur le discours en quête d'acceptabilité diffusé à travers les différents documents émanant des entreprises, vision statement, code of good practices, code of good conduct, etc. Jusqu'ici, nous avons analysé le discours relatif à la quête de l'honneur perdu de l'industrie des jeux du hasard (Isani, 2007a, 2007b) et de «la littérature adulte» (Isani, 2010) aux États-Unis, ainsi que le contre discours émis par les opposants. Notre analyse porte à présent sur le secteur de l'alcool et plus particulièrement sur la célèbre liqueur verte des Alpes françaises, la Chartreuse verte.

2 À cet égard, il convient de souligner un paradoxe fondamental qui définit les cultures occidentales d'aujourd'hui : plus nos sociétés modernes semblent vouloir s'affranchir des tabous d'antan, plus elles se créent de nouveaux secteurs d'activité commerciale à mettre à l'index. Si de nombreuses cultures s'accordent pour considérer l'alcool, le sexe et les jeux de hasard - ce qu'Atherton appelle «the holy trinity of drinking, sex, and gambling " (2006, p. 97) - comme des secteurs d'activité moralement inacceptables, aujourd'hui, la notion de «vice ", associée à des activités commerciales, s'est étendue à de nombreux 
autres secteurs comme la restauration rapide, les armes, le nucléaire, la banque, l'industrie pharmaceutique, voire l'assurance... (Isani, 2007a)

3 La présente étude se démarque des travaux précédents en ce que l'analyse ne porte pas exclusivement sur le discours promotionnel élaboré et mis en place par les entreprises elles-mêmes, mais également sur celui qui apparait, de manière tout à fait inopinée, dans un contexte fictionnel différent de son contexte d'origine pour se transformer en discours de facto promotionnel. L'analyse se situe dans une perspective interculturelle où il s'agit d'examiner comment une brève réplique provenant d'un film américain a pu transformer la Chartreuse verte ${ }^{1}$, une liqueur française connue pour ses vertus médicinales depuis le Moyen Âge, en cocktail « branché » de la jeunesse française.

\section{Quelques propos liminaires...}

4 Avant d'aborder le volet fictionnel de cette analyse, nous présentons brièvement un cadrage factuel pour, dans un premier temps, rappeler les discours antonymiques que suscite le sujet de l'alcool en général, et ensuite fournir quelques indications relatives au comportement de la jeunesse française eu égard à l'alcool.

\subsection{Discours antonymiques}

5 La consommation d'alcool est un sujet de grande ambivalence qui a toujours fait l'objet d'un discours sociétal contradictoire, oscillant entre la promotion et la sanction, de même que les jeux de hasard (Atherton, 2006). Comme de nombreux alcools fabriqués dans des monastères partout dans le monde, la Chartreuse verte, conçue et, pendant longtemps, commercialisée par les Pères chartreux, véhicule un discours promotionnel construit autour du triptyque " secret, sainteté et santé ", à mettre en parallèle avec celui émis par un État protecteur, voire paternaliste, qui met en avant les effets nuisibles du produit dans sa lutte contre l'alcoolisme.

\subsubsection{Discours officiel de dissuasion}

6 En France, le discours officiel de dissuasion concernant l'alcool est riche, abondant et multidisciplinaire (droit, médecine, psychologie, sécurité, éducation, etc.), mais dans le cadre de cette étude, nous nous limiterons à quelques repères législatifs forts relatifs à la consommation d'alcool et à la jeunesse.

7 Comme c'est le cas pour tous les produits de consommation qui ne sont pas « neutres », le législateur s'est intéressé à l'alcool, soit pour réglementer son commerce, soit à des fins fiscales, ou encore pour des raisons de santé publique, voire d'ordre public. À cet effet, la première loi en France règlementant la consommation d'alcool date de 1873 et avait pour objet de sanctionner l'ivresse publique. Pour ce qui concerne la consommation d'alcool et les jeunes, nous retenons deux dates clés : celle de la première législation limitant le support de la publicité pour les boissons alcoolisées, l'amendement Barrot de 1949, qui interdit la publicité pour la bière dans les publications destinées aux jeunes, et puis la dernière en date, la loi Bachelot de 2009 interdisant la vente d'alcool aux jeunes de moins de 18 ans.

8 Entre les deux, la France s'est dotée de la loi marquante du 10 janvier 1991, dite « loi Évin », qui non seulement réglemente les lieux de consommation du tabac mais, en plus, 
soumet le contenu des publicités pour l'alcool à des indications techniques (titre, contenu, couleur, etc.) :

La publicité autorisée pour les boissons alcooliques est limitée à l'indication du degré volumique d'alcool, de l'origine, de la dénomination, de la composition du produit, du nom et de l'adresse du fabricant, des agents et des dépositaires ainsi que du mode d'élaboration, des modalités de vente et du mode de consommation du produit. Cette publicité peut comporter des références relatives aux terroirs de production, aux distinctions obtenues, aux appellations d'origine telles que définies à l'article L. 115-1 du code de la consommation ou aux indications géographiques telles que définies dans les conventions et traités internationaux régulièrement ratifiés. Elle peut également comporter des références objectives relatives à la couleur et aux caractéristiques olfactives et gustatives du produit. (Code de la santé publique, article 3323-4, loi du 10 janvier 1991 relative à la lutte contre le tabagisme et l'alcoolisme.)

Elle exige également l'affichage d'une mise en garde contre les dangers de l'alcool : Toute publicité en faveur de boissons alcooliques, à l'exception des circulaires commerciales destinées aux personnes agissant à titre professionnel ou faisant l'objet d'envois nominatifs ainsi que les affichettes, tarifs, menus ou objets à l'intérieur des lieux de vente à caractère spécialisé, doit être assortie d'un message de caractère sanitaire précisant que l'abus d'alcool est dangereux pour la santé. (Code de la santé publique, article 3323-4, loi du 10 janvier 1991 relative à la lutte contre le tabagisme et l'alcoolisme.) [Nos italiques]

\subsubsection{Discours promotionnel séculaire : « secret, sainteté et santé »}

9 L'avertissement ciblant le produit comme dangereux pour la santé est une première entorse au discours promotionnel valorisant les vertus médicinales du produit qui émanait des premiers moines distillateurs eux-mêmes pour qui «l'intérêt n'est pas tourné vers la pierre philosophale, mais porte sur l'élixir de longue vie, le remède parfait » (Steinmetz, 2006, p. 14). L'élixir à ingrédients mystérieux fabriqué par les Pères chartreux, titre environ $70^{\circ}$ et est, à l'origine, un produit conçu, commercialisé et consommé uniquement à des fins médicinales, comme l'indiquent des étiquettes encore relativement récentes (avant 1930) que l'on peut trouver sur le site < www.delachartreuse.blogspot.fr> : "L'Élixir végétal, souverain contre les indigestions, maux d'estomac, syncopes, influenza, choléra, mal de mer », ou encore, «L'Élixir végétal, cordial puissant, digestif remarquable. Indispensable aux familles. En vente dans toutes les bonnes épiceries, pharmacies, etc.». Ce discours promotionnel, fondé sur les vertus médicinales du produit, se poursuit avec la fabrication en 1764 de la célèbre Chartreuse verte, commercialisée comme « liqueur de santé ».

10 En 1970, les moines confient la commercialisation de la liqueur à une entreprise créée à cette fin, la société Chartreuse Diffusion qui se situe à Voiron, non loin du monastère où continuent à vivre et à travailler les moines chartreux. Le discours promotionnel émanant de l'entreprise liquoriste poursuit la ligne axée sur le discours traditionnel de « secret, sainteté et santé »... jusqu'à l'avènement de la loi Évin de 1991. C'est alors que le volet « santé » du discours promotionnel se voit obligé de s'effacer et que le volet « secret et sainteté » devient désormais l'élément identitaire fort de la marque, aux niveaux explicite et implicite.

11 Au niveau du discours explicite, le récit affiché sur le site web de l'entreprise tisse le lien indissociable entre les moines, la liqueur et le secret de sa fabrication à travers les aléas de l'histoire depuis 1064 jusqu'à nos jours. L'implication monacale est mise en valeur par 
un renvoi patronymique aux deux moines distillateurs qui connaissent le secret de la fabrication, et le lieu sacré qu'est le monastère de cet ordre de silence et de secret situé dans le massif de la Grande Chartreuse. Sont aussi soulignées les retombées spirituelles que peut apporter ce qui pour certains pourrait ne représenter que la simple commercialisation d'un alcool :

Seuls les Pères chartreux connaissent les noms des 130 plantes utilisées pour faire la liqueur Chartreuse ; 2 chartreux, Dom Benoit et Frère Jean-Jacques, sont en charge de la fabrication.

Les 18 tonnes de plantes nécessaires chaque année sont livrées par différents fournisseurs au monastère de la Grande Chartreuse à St-Pierre-de-Chartreuse. La «salle des plantes » se trouve dans l'ancienne boulangerie du monastère; là, les plantes séchées sont triées, broyées, pesées et mélangées selon la recette de 1605. Puis, elles sont livrées à la distillerie de Voiron dans des grands sacs numérotés. [...] Toutes ces liqueurs basées sur la recette d'un grimoire du $16^{\mathrm{e}}$ ou $17^{\mathrm{e}}$ siècle ont été élaborées par les Pères chartreux; leur commercialisation permet à leur communauté de survivre et de continuer à prier dans le silence et la solitude, selon l'inspiration de saint Bruno. (<www.chartreuse.fr/la-fabrication-aujourdhui;article; 66;fr.html>, consulté le 28 janvier 2014)

12 Au niveau de l'implicite, le discours de la sémiotique visuelle renforce la thématique de la « sainteté » en focalisant sur les origines « ecclésiastiques » de la liqueur à travers le logo de l'entreprise où la croix et l'orbe empruntées aux armoiries de l'ordre monacal flottent au centre de chaque étiquette, ainsi que sur les nombreuses images présentes dans les locaux ouverts au public, et reprises, à leur tour, à l'infini par les internautes : un moine scrutant "l'or vert» dans une éprouvette ou, plus puissante car plus évocatrice du mystique religieux, la silhouette d'un moine en robe et capuche blanches parmi les alambics et les foudres de vieillissement.

Si le diptyque discursif du « secret et sainteté » reste inchangé, le volet « santé » évolue, à travers une textualité en mode déclaratif, vers le domaine connexe de la "nature ", atout inestimable car intégrateur positif. Ainsi, sur un fond vert, couleur emblématique à la fois de la liqueur et de la Nature, est mis en avant le fait que la fabrication de la liqueur requiert 18 tonnes annuelles des 130 herbes et plantes naturelles qui entrent dans la composition de la liqueur, et que le tout macère dans un alcool de raisin - que du naturel. Soin est pris de souligner que même la couleur sui generis de la Chartreuse verte est entièrement naturelle, comme l'explique le site spécialisé L'Artisanat Monastique: "[...] la Chartreuse verte est la seule liqueur verte naturelle jusque dans sa couleur (chlorophylle).»

14 À ce discours textuel s'ajoute ce que nous avons appelé ailleurs le discours actionnel (Isani, 2007a) selon lequel une entreprise transpose en actes les objectifs véhiculés par les discours de promotion textuel et visuel. Dans cet effort, visant à rattacher le volet « perdu » de la santé à celui de la Nature, la société liquoriste a entrepris un mécénat qui se définit autour du thème de la montagne, thématique qui rapproche ainsi le produit de son lieu d'ancrage identitaire, le sanctuaire monacal du Désert de la Grande Chartreuse, un lieu millénaire à la fois secret, saint et sain.

Il est à noter cependant que, si depuis 1991, le discours de la "santé " émanant des moines distillateurs a dû céder la place au discours dissuasif de la « santé » émanant de l'État, le discours jussif de dissuasion, pour aussi officielle qu'en soit l'origine, ne semble ni avoir entamé le discours jouissif des consommateurs, ni modifié la langue française, comme en témoigne la proposition encore d'actualité aujourd'hui après un bon dîner: «Un petit digestif?» 


\subsection{Comportements de consommation d'alcool des jeunes en France}

Confortée par des reportages sur le binge drinking au Royaume-Uni, ou el botellón y los jovenes en Espagne, par des images choc de publicités visant à sensibiliser les jeunes conducteurs des dangers de la conduite en état d'ivresse, ou encore les images des supporteurs des clubs de foot lors des matchs, l'impression générale est que les jeunes d'aujourd'hui consomment beaucoup plus d'alcool que ceux des générations précédentes. Pourtant, les statistiques présentent un tableau plus nuancé qui met l'accent sur un changement dans les comportements de consommation plutôt que sur une augmentation des quantités consommées. Comme le précisent Freyssiney-Dominjon et Wagner dans leur étude L'alcool en fête: manière de boire de la nouvelle jeunesse étudiante : "On connaît la spécificité du boire des jeunes. Ils boivent moins fréquemment que leurs aînés, et ils sont plus souvent sujets à l'ivresse. » (2003, p. 1)

Dans la même lignée, l'OFDT (Observatoire français des drogues et de la toxicomanie) et l'association Entreprise et Prévention (Producteurs de boissons pour la prévention du risque alcool) s'accordent sur deux constats: il y a une forte diminution de la consommation d'alcool en France depuis les années 1950, et la fréquence de consommation quotidienne routinière diminue en faveur d'une fréquence hebdomadaire festive, comme l'indique la figure ci-dessous, reproduite d'un article du Figaro en ligne du 4 novembre 2013, intitulé « Les Français boivent toujours trop » :

Figure 1. - Consommation d'alcool en baisse.

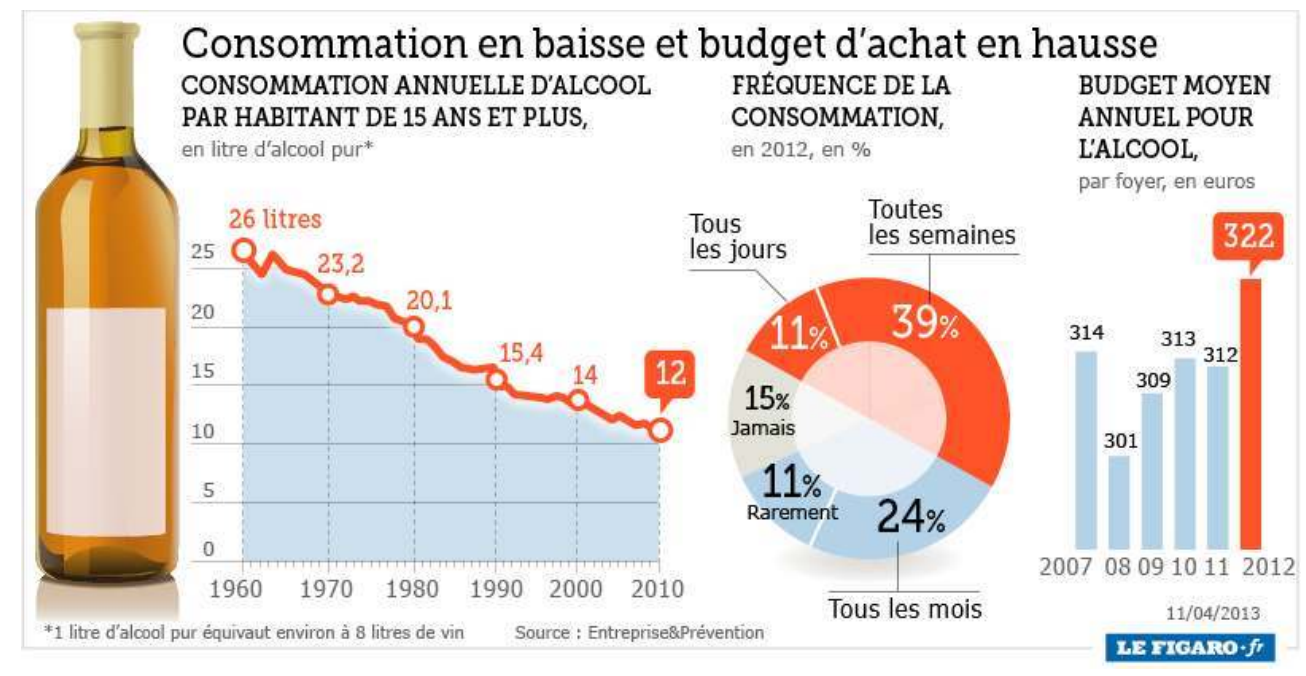

Le Figaro en ligne, 4 novembre 2013

(<www.lefigaro.fr/actualite-france/2013/04/11/01016-20130411ARTFIG00504-les-francais-boiventtoujours-trop.php>, consulté le 24 janvier 2014).

D'autres résultats d'enquêtes publiés par l'OFDT mettent en lumière d'autres tendances relatives à la consommation d'alcool des jeunes en France qui nous intéressent dans le cadre de cette étude, comme l'indique la figure ci-dessous : 
Figure 2. - Types et quantités d'alcools consommés par les jeunes.

Quantité d'alcool consommé par habitant âgé de 15 ans et plus depuis 1961 (en litres équivalents d'alcool pur)

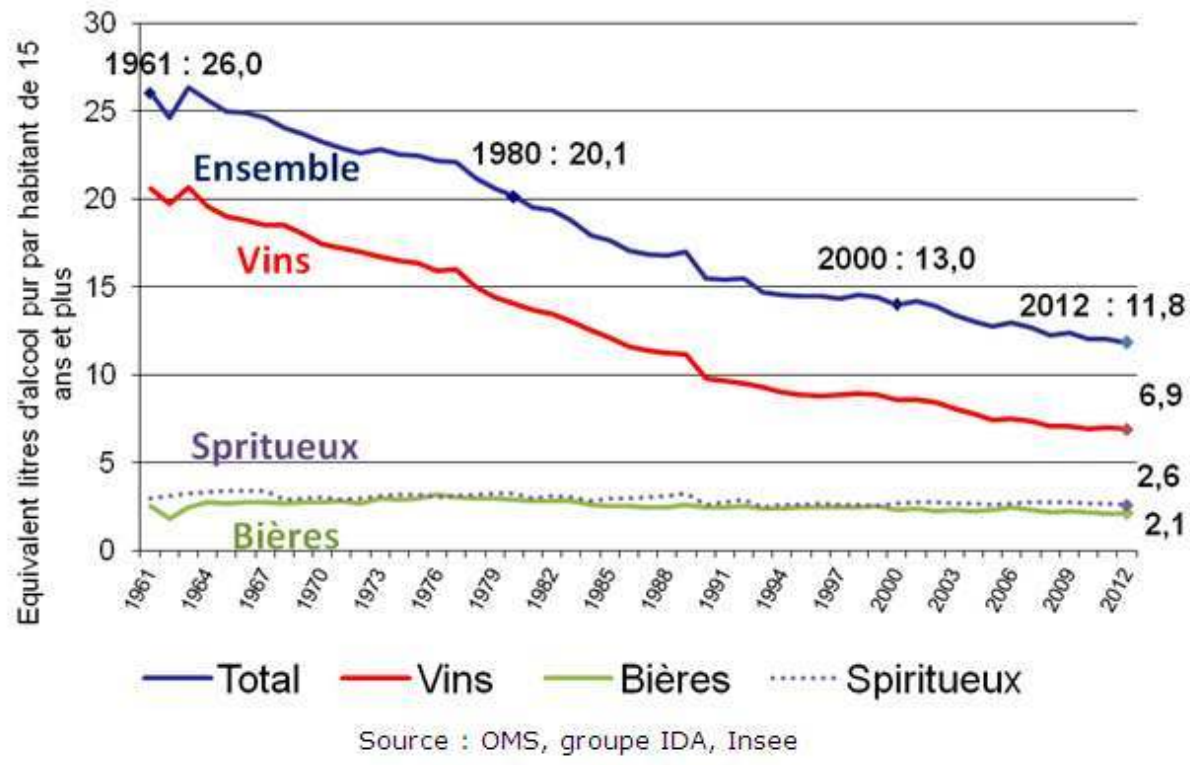

Le Figaro en ligne, 4 novembre 2013

(<www.ofdt.fr/BDD_len/seristat/00014.xhtml>, consulté le 28 janvier 2014)

Pour résumer ces données chiffrées, nous retiendrons de la lecture de ces données que si la consommation dans son ensemble et celle du vin en France diminuent auprès des jeunes, celle de la bière et des spiritueux reste stable, ces dernières représentant les catégories les plus consommées par les jeunes.

\section{Le cinéma américain : moteur promotionnel inopiné de la Chartreuse verte}

Nous en venons maintenant au cœur de notre problématique, à savoir, comment un heureux hasard de mise en fiction - un authentique cas de séréndipité - peut s'avérer un moteur promotionnel bien plus puissant qu'un discours promotionnel, recherché, planifié, et dispendieux.

21 La fiction anglophone a rendu plusieurs hommages à la Chartreuse qui est mentionnée en passant dans de nombreux romans. Il existe, en particulier, deux exemples dans la littérature britannique datant de la première moitié $\mathrm{du} \mathrm{xx}^{\mathrm{e}}$ siècle qui peuvent, encore aujourd'hui, être considérés comme les hommages littéraires les plus mémorables rendus à la liqueur verte. La première référence revient à $\mathrm{H}$. H. Munro (nom de plume Saki) et auteur d'un récit datant de 1904 intitulé Reginald On Christmas Presents, où il évoque la question habituelle de la difficulté à choisir des cadeaux de Noël appropriés :

Personally, I can't see where the difficulty in choosing suitable presents lies. No boy who had brought himself up properly could fail to appreciate one of those decorative bottles of liqueurs that are so reverently staged in Morel's window-and 
it wouldn't in the least matter if one did get duplicates. And there would always be the supreme moment of dreadful uncertainty whether it was crème de menthe or chartreuse-like the expectant thrill of seeing your partner's hand turned up at bridge. People may say what they like about the decay of Christianity; the religious system that produced Green Chartreuse can never really die. (1993 [1904], p. 6-7) [Nos italiques]

Il faudra attendre quarante ans avant que la Chartreuse verte ne marque à nouveau l'imaginaire d'un auteur britannique. Il s'agit du roman Brideshead Revisited écrit en 1945 par l'auteur Evelyn Waugh, adapté pour la télévision en 1981 et pour le cinéma en 2008. L'un des thèmes du roman est l'alcoolisme des jeunes étudiants à l'université d'Oxford, dont l'un des plus insondables, Anthony Blanche, évoque la Chartreuse verte en filant une belle métaphore synesthésique :

Anthony had lost his stammer in the deep waters of his old romance. It came floating back to him, momentarily, with the coffee and liqueurs. "Real G-g-green Chartreuse, made before the expulsion of the monks. There are five distinct tastes as it trickles over the tongue. It is like swallowing a spectrum." $(1945$, p. 63) [Nos italiques]

De l'autre côté de l'Atlantique, la Chartreuse verte entre également dans l'imaginaire auctorial des romanciers américains pour véhiculer l'image de personnages et de lieux quelque peu hors de l'ordinaire. C'est le cas dans le roman The Great Gatsby écrit en 1925 by F. Scott Fitzgerald, où la fin de la visite de la somptueuse demeure de Gatsby se termine par un verre de Chartreuse - «Finally we came to Gatsby's own apartment, a bedroom and a bath, and an Adam study, where we sat down and drank a glass of some Chartreuse he took from a cupboard in the wall» (p. 72) - un spiritueux bien plus en phase que ne l'aurait été le whisky avec l'image que le protagoniste cherche désespérément à cultiver.

Pour Claire Dixsaut, auteure du site Cinémiam, c'est à partir de ce moment que «la sorcière verte » fait une entrée remarquée aux États-Unis :

Et puis tout s'accélère. La liqueur apparaît dans d'énormes best-sellers : Gatsby le Magnifique et Retour à Brideshead, écrits respectivement par F. Scott Fitzgerald et Evelyn Waugh, de grands connaisseurs de boissons. Drink de l'élite, boisson clandestine, la chartreuse devient la favorite des chefs du Milieu américain. ( La sorcière verte »: <http://cinemiam.com/2012/05/18/chartreuse-de-la-mort/>, consulté le 28 janvier 2014)

Cependant, force est de constater que, malgré la finesse de ces évocations littéraires, ce n'est pas cette catégorie de mise en fiction qui est susceptible de dynamiser l'intérêt de la jeunesse, qu'elle soit française ou autre. Le véritable déclencheur du phénomène se situe à une période plus actuelle et se trouve dans un moyen de diffusion infiniment plus puissant que le roman: le film Death Proof (Boulevard de la mort) réalisé par Quentin Tarantino en 2007.

Il s'agit alors d'un changement radical de décor et de registre abandonnant toute velléité de discours visant à promouvoir la liqueur en termes de "secret, sainteté et santé ", de mystère, d'élégance et de raffinement: les films de Tarantino ${ }^{4}$ sont connus pour leur extrême violence, leurs dialogues "déjantés » et outranciers, leur extravagance en tout genre, leurs multiples et complexes références à la culture cinématographique, leur humour, leurs excellents comédiens, leur anti-américanisme explicite et leur proeuropéanisme implicite, etc. Ce sont aussi des films cultes pour toute une génération âgée 18 à 35 ans, l'audience cible de Tarantino.

Dans Boulevard de la mort ${ }^{5}$, la présence de la Chartreuse verte n'est pas réduite à un simple accessoire servant à planter le décor, mais constitue le sujet d'une séquence (< www.youtube.com/watch?v=0Tw5lvr7afM $>$ ) construite autour d'un échange qui se 
termine par la réplique clé de douze mots - "Chartreuse, the only liqueur so good they named a color after $i t^{6} »-$ une réplique, désormais mythique, qui renvoie à deux plans référentiels différents, la boisson et la couleur ${ }^{7}$.

Cette séquence, mise en valeur par le fait que c'est Tarantino lui-même qui joue le rôle du barman faisant l'éloge de cette liqueur jusqu'ici relativement obscure, constitue la deuxième entorse au discours promotionnel traditionnel car, pour aussi valorisante qu'elle soit pour la Chartreuse, elle apparait au milieu d'une scène d'ivresse débridée et tapageuse qui contraste avec l'image feutrée du digestif monastique dégusté à petites gorgées. C'est cette réplique qui déclenche un véritable engouement pour la Chartreuse verte auprès d'une nouvelle génération de consommateurs, tout d'abord aux États-Unis et ensuite en France.

\section{Une réplique de douze mots : quelques retombées...}

Les retombées de ce discours promotionnel inattendu provenant d'une réplique de 12 mots se sont fait ressentir dans plus d'un domaine et dans plus d'un pays. Nous proposons, tout d'abord, d'analyser brièvement quelques-unes des retombées pour la première partie prenante concernée, l'entreprise elle-même, avant de chercher à mesurer l'engouement pour la Chartreuse verte aux États-Unis et puis en France.

Si mesurer les retombées commerciales pour l'entreprise relève d'un domaine aisément vérifiable à travers la diffusion de chiffres officiels, apprécier les effets sociétaux s'avère plus complexe : comment évaluer les dires concernant la transformation de la Chartreuse verte, jadis liqueur digestive un peu surannée, en cocktail tendance de la jeunesse branchée? Idéalement, nous aurions souhaité disposer, en un clic, de statistiques directes, chiffrées et fiables démontrant que de telle période à telle période, les jeunes de telle fourchette d'âge ne touchaient pas à cette liqueur et que c'est à partir de l'année $\mathrm{A}$ qu'ils en ont fait un breuvage fétiche en achetant tant de bouteilles par an. Malheureusement, des statistiques de ce genre n'existent pas dans un domaine aussi sensible et complexe que celui de la consommation d'alcool.

31 Au vu de cela, nous avons entrepris de compléter une approche chiffrée par des données obliques, indirectes. La première démarche a consisté à recueillir des données chiffrées relatives à l'intérêt généré par la Chartreuse par rapport à d'autres spiritueux français de la même catégorie sur l'Internet; la deuxième démarche abandonne l'approche chiffrée en faveur d'une approche davantage sociologique qui cherche à se renseigner à partir de l'observation et l'analyse in situ, en se rendant sur le terrain, en visitant les lieux, en interrogeant les acteurs et en échangeant avec d'autres parties prenantes. Il va de soi que ce type d'approche laisse la porte ouverte aux critiques portant sur l'absence de données quantitatives, la nature informelle des données ainsi recueillies, la subjectivité du recueil et de l'interprétation des données, les échantillonnages limités, le biais du chercheur, etc. Néanmoins, il n'en est pas moins vrai que dans les contextes touchant à l'humain, le recueil de « hard data » n'est pas toujours l'approche la plus appropriée et que l'analyse qualitative proposée par l'approche ethnographique permet d'établir une convergence d'indices permettant d'identifier des tendances et d'en dégager les grandes lignes. 


\subsection{Retombées commerciales}

En 1970 les moines chartreux confient la commercialisation de leur produit à la société voironnaise, Chartreuse Diffusion, en échange d'un versement de royalties au monastère. Naturellement, cette entreprise a été la première à bénéficier des retombées favorables de cette réplique de douze mots, comme en témoigne la figure suivante présentant l'évolution de son chiffre d'affaires au cours des six dernières années :

Figure 3. - Chartreuse Diffusion : évolution du chiffre d'affaires (en milliers d'euros).

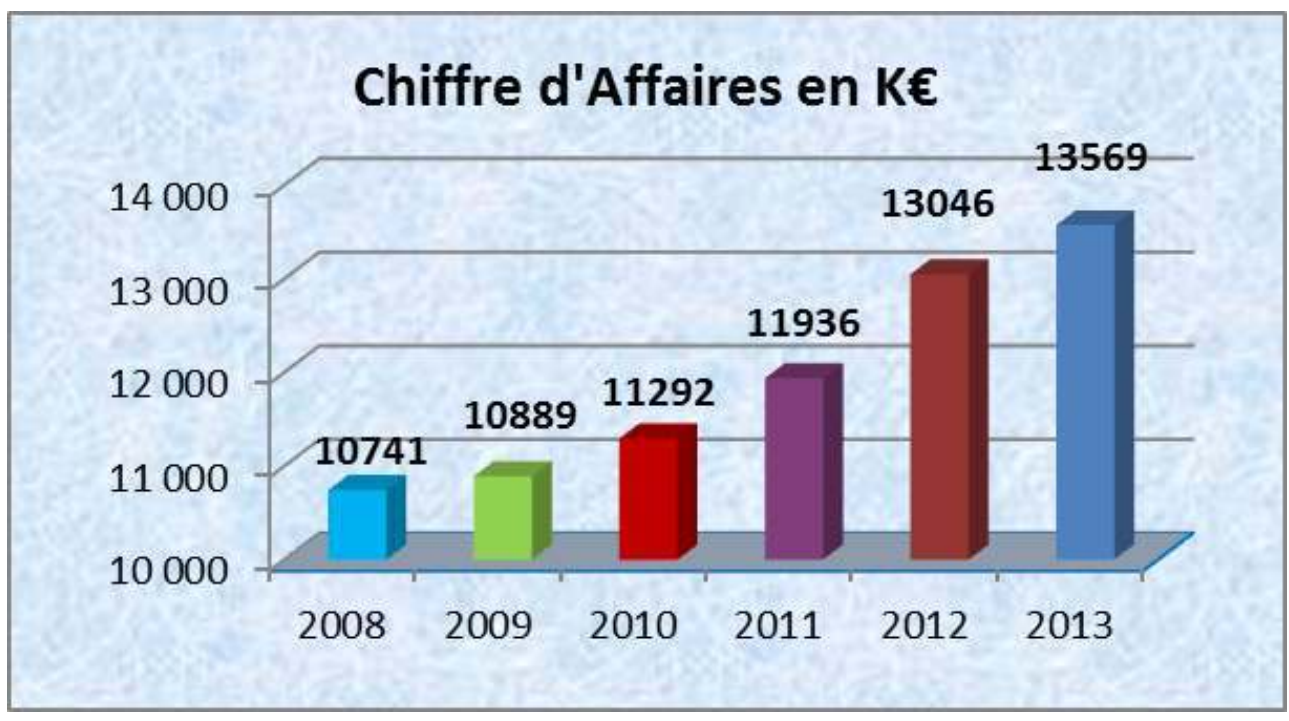

Source des données chiffrées : <https://www.score3.fr/bilans-et-ratios-financiers.shtml\#comptes> (consulté le 18 février 2014).

Sans être des spécialistes de l'analyse financière, nous pouvons constater que depuis 2008 (rappelons ici que le film est sorti en 2007), le chiffre d'affaires de la société est en augmentation constante de $4 \%$ à $5 \%$ par an avec une hausse record enregistré pour 2011, année où les ventes ont augmenté de plus de $10 \%$. Ces bons chiffres sont à mettre en corrélation avec un taux de croissance des bénéfices exponentiel, comme le montre l'histogramme ci-après : 
Figure 4. - Chartreuse Diffusion : évolution des bénéfices (en milliers d'euros).

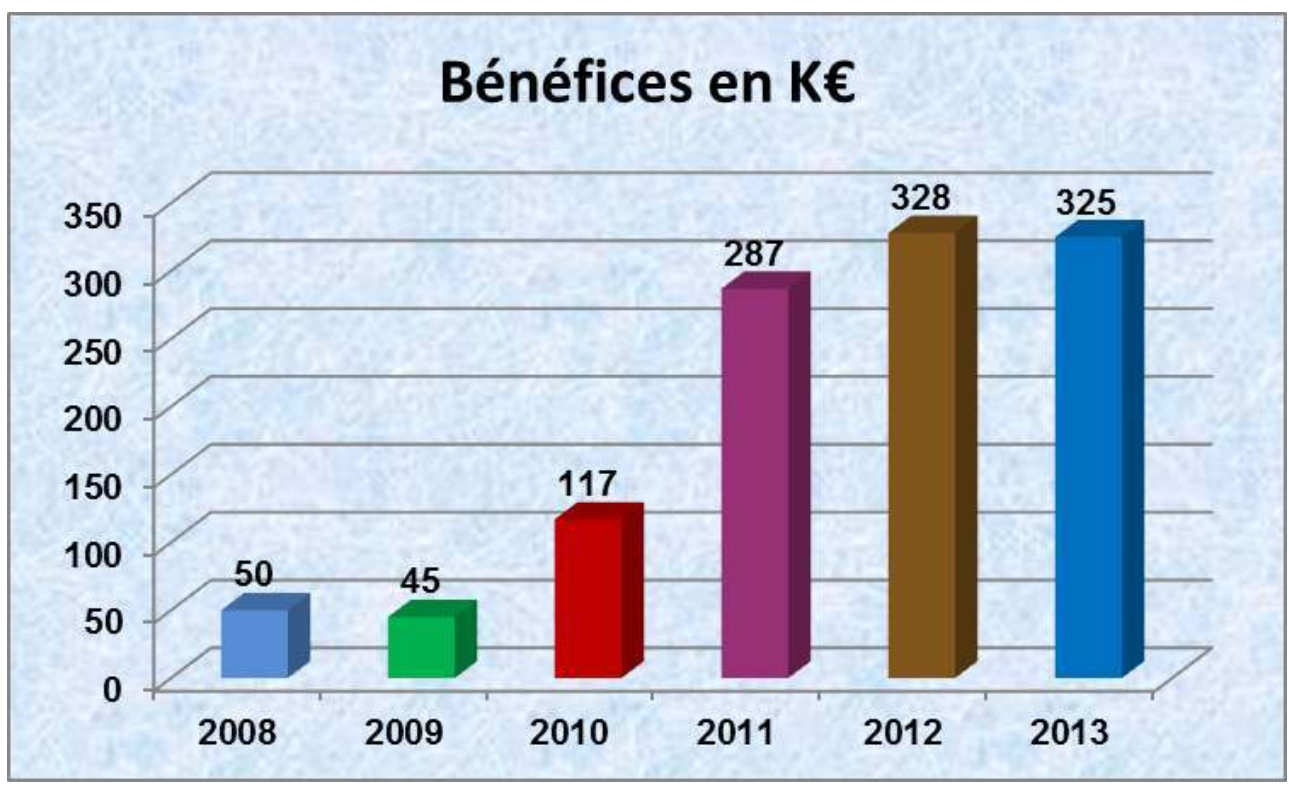

Source des données chiffrées : <https://www.score3.fr/bilans-et-ratios-financiers.shtml\#comptes> (consulté le 18 février 2014).

L'évolution très positive de ces résultats prend toute sa valeur lorsqu'elle est mise en regard avec la récession mondiale qui sévit pendant cette période et dont l'origine se trouve dans la crise des subprimes aux États-Unis qui a commencé en... 2008.

\subsection{Engouement pour la Chartreuse aux États-Unis}

Les premières retombées du film Boulevard de la mort, film fait par un Américain pour un public essentiellement américain, se sont naturellement fait sentir tout d'abord aux ÉtatsUnis. Le phénomène est étayé par les éléments suivants.

\subsubsection{Chiffres à l'exportation vers le marché américain}

Le lien entre le film et l'engouement pour la Chartreuse auprès du public américain peut être évalué par les chiffres d'exportation de la société Chartreuse Diffusion. Depuis 2011, les exportations de la Chartreuse se sont envolées (+10\%), avec $50 \%$ de la production destinée aux marchés étrangers dont $50 \%$ se destine au seul marché des États-Unis. Les États-Unis sont désormais le premier pays importateur de la liqueur, position jusqu'ici réservée à l'Espagne.

\subsubsection{Statistiques quantitatives: moteur de recherche anglophone}

Pour compléter les données commerciales, nous avons recherché des données statistiques et quantitatives portant sur le «Internet chatter ", soit les échanges générés au sujet du produit sur l'Internet.

Nous avons cherché à comparer l'intérêt pour la Chartreuse sur l'Internet à l'intérêt pour d'autres spiritueux français de la même catégorie (prix, titrage, mode de consommation, popularité, image, etc.), notre choix s'arrêtant sur deux produits qui nous paraissaient 
présenter des similarités en termes d'image, le Cognac et le Cointreau. La procédure utilisée pour obtenir des données statistiques était simple et consistait à mesurer le nombre de résultats ou « hits » obtenus pour chacun des trois produits à travers une série de trois recherches effectuées à partir d'une formule d'entrée variable à chaque fois.

Dans un premier temps, nous avons cherché à obtenir des résultats d'ordre général à une échelle internationale à partir d'une entrée très peu discriminante, [cocktail + nom de l'alcool], dans le moteur de recherche Google.com. Nous avons privilégié le terme «cocktail» tout d'abord parce qu'il correspond à l'usage prédominant de la liqueur aujourd'hui, mais également parce qu'il s'agit d'un terme translangue qui permettait de situer la recherche à un niveau international et en même temps, de limiter les références aux entités géographiques affiliées à " Cognac » et à « Chartreuse » qui ont sérieusement parasité les premières recherches :

Figure 5. - Résultats obtenus pour [cocktail + nom de l'alcool].

\begin{tabular}{|ll|}
\hline Cognac & 4690000 \\
Cointreau & 1310000 \\
Chartreuse & 1050000 \\
\hline
\end{tabular}

Google.com (27 janvier 2014)

40 Comme nous aurions pu nous y attendre, le Cognac, alcool dont l'éponymisation est un phénomène translangue, vient en premier, le Cointreau, une liqueur qui jouit, à l'étranger, d'une notoriété plus ancienne que la Chartreuse, en deuxième, et la Chartreuse en troisième.

41 L'objet de la deuxième recherche consistait à cerner de plus près l'intérêt porté à la Chartreuse dans les pays anglophones, plus particulièrement aux États-Unis ${ }^{8}$. La recherche a été lancée avec une formule d'entrée plus discriminante sur le plan linguistique et culturel, [cocktail + recipe], recipe signifiant recette en anglais.

Figure 6. - Résultats obtenus pour [cocktail + recipe].

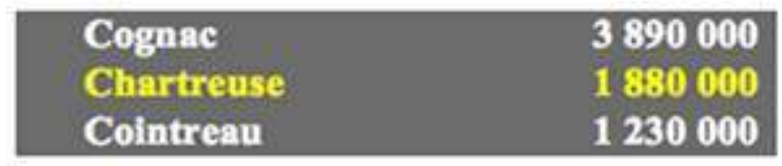

Google.com (27 janvier 2014)

Comme on peut le voir, les résultats de cette recherche situent la Chartreuse en $2^{\mathrm{e}}$ position, permettant de penser que la Chartreuse génère désormais plus d'intérêt dans le milieu des mixologistes anglophones que le Cointreau, liqueur plus connue et plus traditionnelle dans la fabrication des cocktails.

Une $3^{\mathrm{e}}$ recherche a eu pour but d'approfondir la question en mesurant l'intérêt porté à ces liqueurs en tant que produit à la mode. Pour ce faire, la formule d'entrée a été modifiée en reformulant le qualificatif variable discriminant pour aboutir à [cocktail + « in cocktail »]", signifiant « cocktail branché » en anglais. 
Figure 7. - Résultats obtenus pour [nom + « in cocktail »].

\begin{tabular}{|ll|}
\hline Chartreuse & $\mathbf{4 3 9 0 0}$ \\
Cognac & 17100 \\
Cointreau & 13500 \\
\hline
\end{tabular}

Google.com (27 janvier 2014) film Boulevard de la mort, il y a eu un engouement mesurable d'une certaine catégorie socio-économique de la population américaine pour la liqueur verte, nous nous tournons maintenant vers le même phénomène en France que nous abordons avec la même approche heuristique.

\subsubsection{Quelques chiffres}

Le renouveau de la Chartreuse verte réside essentiellement dans l'évolution des perceptions du consommateur concernant son usage. Cantonnée jadis au rôle de digestif et donc à consommer dans un cadre socio-temporel spécifique, elle est aujourd'hui appréciée comme un ingrédient de cocktail à usages multiples qui se consomme partout et à de nombreux moments de la journée. L'entreprise elle-même, tout en prenant soin de préserver son statut d'origine en tant que digestif, met en avant cette évolution: «Traditionnellement, elle est considérée comme un digestif, mais de plus en plus de connaisseurs la dégustent aussi en cocktails.» (<www.chartreuse.fr/chartreuseverte;fiche;3;fr.html>, consulté le 28 janvier 2014)

Il existe de nombreuses études sur la consommation de l'alcool, mais celle publiée par Nielson-CGA en 2013 attire particulièrement l'attention puisqu'elle porte sur la «culture de cocktails » qui s'instaure en France :

D'après la dernière étude réalisée par Nielsen et CGA sur la place du cocktail en Cafés-Hôtels-Restaurants (CHR), il apparaît que plus d'1 établissement du CHR sur 3 propose une offre cocktail à ses clients. Plus qu'un phénomène de mode, le cocktail s'inscrit comme une tendance de consommation pérenne. Plus de la moitié des Français âgés de 18 à 60 ans consomme des cocktails avec ou sans alcool en CHR.

Si l'on ajoute la consommation de cocktail à domicile, c'est plus de 3 Français sur 4 qui sont consommateurs de cocktail. Cette tendance de consommation est plus forte chez les jeunes ce qui laisse à penser que le cocktail a le vent en poupe pour les années à venir. L'étude Nielson-CGA révèle une réelle démocratisation du mode de consommation des cocktails en France. Le cocktail est en effet sorti des hôtels 4-5 étoiles auxquels on avait l'habitude de l'associer un peu trop systématiquement. (<www.nielsen.com/fr/fr/news-insights/press-releases/2013/nielsen-cga-lemojito-en-tete-dans-les-cafes-hotels-restaurant.print.html\#sthash.EfJXaEnN.dpuf>, consulté le 19 février 2014) 


\subsubsection{Données de type sociologique}

Pour aller plus loin dans la validation des impressions, nous avons recouru à une enquête à partir d'entretiens avec les acteurs concernés ainsi que d'observation et d'analyse in situ . Tout d'abord, nous avons exploité le vivier que représentent nos étudiants pour nous renseigner sur les habitudes et les goûts de deux groupes d'une vingtaine étudiants qui se définissaient comme des «fêtards » en deuxième année de Master (promotions 2011 et 2012) quant à leurs habitudes et goûts par rapport à la consommation de la Chartreuse. Le croisement des renseignements ainsi obtenus fait ressortir les traits communs suivants :

Figure 8. - Informations obtenues à partir d'entretiens informels.

\begin{tabular}{|c|c|}
\hline Concernant leur consommation d'alcool... & Concernant la Chartreuse... \\
\hline $\begin{array}{l}\text { - Ils consomment plus à la maison qu'à l'extérieur. } \\
\text { - Quand ils consomment à l'extérieur, c'est dans des } \\
\text { bars proposant l'Happy Hour et à cette occasion, ils } \\
\text { consomment des cocktails. } \\
\text { - Ce sont les garçons qui se considèrent comme les } \\
\text { spécialistes du nouvel art de la «mixologie ". } \\
\text { - Ils aiment faire des cocktails pour leurs fêtes. } \\
\text { - Pour les fêtes à grand nombre, les cocktails les plus } \\
\text { courants sont le Mojito, le Cuba libre, la soupe } \\
\text { angevine, le ponch et le whisky Coca, } \\
\text { essentiellement parce que les ingrédients sont } \\
\text { moins chers. }\end{array}$ & $\begin{array}{l}\text { - Des deux Chartreuses, la verte et le } \\
\text { jaune, la verte est préférée. } \\
\text { - La Chartreuse est davantage consommée } \\
\text { sous forme de cocktails que de digestif. } \\
\text { - Les cocktails à base de Chartreuse verte } \\
\text { sont parmi les plus recherchés mais, vu le } \\
\text { prix de la Chartreuse, ils sont réservés } \\
\text { pour des fêtes en petit comité. } \\
\text { - En dehors des cocktails classiques, le } \\
\text { Green chaud (chocolat chaud + Chartreuse } \\
\text { verte) est une boisson très appréciée }{ }^{10} \text {. }\end{array}$ \\
\hline
\end{tabular}

Dans un deuxième temps, en 2012, nous avons interviewé deux étudiantes qui travaillaient dans deux pubs du centre-ville et qui ont confirmé que la Chartreuse verte figurait effectivement parmi les alcools souvent demandés le soir des week-ends. Dans l'un des établissements, nous avons pu rencontrer le gérant/propriétaire qui nous a confirmé que ses commandes de Chartreuse verte avaient augmenté au cours des trois dernières années, sans pour autant vouloir fournir d'autres précisions.

\subsubsection{Recueil de données par un moteur de recherche francophone}

50 À l'instar de la démarche entreprise pour mesurer l'intérêt suscité par la Chartreuse verte aux États-Unis, nous avons entrepris de mesurer l'intérêt généré sur l'Internet à partir d'entrées linguistiquement et culturellement ciblées vers une population de jeunes francophones. Cette recherche a été faite à partir du moteur de recherche Google.fr.

51 En partant des trois alcools qui ont servi pour la recherche précédente, la première recherche a eu pour objet de cerner l'intérêt général, toute population francophone confondue, à partir de l'entrée [nom + cocktail], et a produit les résultats suivants : 
Figure 9. - Résultats obtenus pour [nom + cocktail].

\begin{tabular}{|ll|}
\hline Cognac & 5640000 \\
Chartreuse & 1800000 \\
Cointreau & 1100000 \\
\hline
\end{tabular}

Google.fr (27 janvier 2014).

52 Comme nous pouvons le constater, si les résultats obtenus pour le Cognac suivent le même schéma que ceux obtenus par la recherche portant sur les États-Unis, ceux relatifs aux deux autres alcools, en revanche, s'inversent, avec la Chartreuse en deuxième position suivie par le Cointreau en troisième.

Pour ce qui concerne les résultats de la recherche suivante à partir de l'entrée [nom + cocktail recette] qui figure ci-dessous, contre toute attente, la position de la Chartreuse semble s'affaiblir par rapport au Cointreau, à l'opposé des résultats obtenus pour les États-Unis où la Chartreuse se situait fermement en position deux. Se pose ici la question de savoir si cela serait éventuellement dû au fait que les Américains ont, davantage que les Français, l'habitude de « publier » leurs recettes de cocktails en ligne...

Figure 10. - Résultats obtenus pour [nom + cocktail recette].

\begin{tabular}{|lr|}
\hline Cognac & 124000 \\
Colntreau & 51000 \\
Chartreuse & 23000 \\
\hline
\end{tabular}

Google.fr (27 janvier 2014).

54 La démarche suivante a cherché à cibler une population plus jeune avec une entrée plus discriminante sur le plan linguistique [nom + « cocktail branché »] :

Figure 11. - Résultats obtenus pour [nom + « cocktail branché »]11

\begin{tabular}{|lr|}
\hline Cognac & 4330 \\
Chartreuse & 3850 \\
Cointreau & 704 \\
\hline
\end{tabular}

Google.fr (27 janvier 2014).

En tant que "cocktail branché ", la Chartreuse retrouve sa deuxième position. En revanche, à l'opposé des résultats obtenus pour les États-Unis, et même si l'écart entre la Chartreuse et le Cointreau se creuse de manière significative, la Chartreuse ne semble pas générer davantage d'intérêt que le Cognac en tant que « cocktail branché » en France.

Au vu de la spécificité de la «culture sport » associée à la Chartreuse verte, notamment dans le cadre de la montagne, berceau originel de la liqueur, et des sports d'hiver, nous avons entrepris une dernière recherche visant à déterminer l'intérêt porté au "Green chaud » (chocolat chaud + Chartreuse verte) par rapport à une autre boisson alcoolisée traditionnellement associées à l'hiver, le vin chaud ${ }^{12}$. 
Figure 12. - Résultats obtenus pour [Green chaud].

\begin{tabular}{|ll|}
\hline Green chaud & 5840000 \\
Vin chaud & 1300000 \\
\hline
\end{tabular}

Google.fr (27 janvier 2014).

57 Les chiffres sont éloquents : le «Green chaud » se positionne de loin avant le vin chaud, boisson plus traditionnelle. Compte tenu du public susceptible d'être attiré par la consommation dans les bars des stations de ski, il nous est permis de supputer que dans le milieu de la montagne et des sports d'hiver, la Chartreuse verte est aujourd'hui un ingrédient qui attire la jeunesse.

$\mathrm{Au}$ vu de ces éléments, il convient de s'interroger sur le paradoxe qui fait qu'une liqueur dont l'image était associée par des jeunes à des consommateurs plutôt âgés, est considérée aujourd'hui, par la même jeunesse, comme un cocktail branché. Quelques éléments de réponse se trouvent peut-être dans le domaine des représentations et des perceptions culturelles et dans l'épineuse question des cultures valorisées et des cultures dévalorisées. Pour illustrer cela, nous recourrons aux travaux du psychologue Abraham Maslow (1954) et à sa pyramide hiérarchique des besoins. Au quatrième et avant dernier rang de sa hiérarchie des besoins, figure le besoin d'estime, l'estime de soi qui se réalise à travers l'hétéro-évaluation positive, le besoin du regard approbateur, voire admiratif, de l'Autre. Il s'ensuit que sentir des éléments de sa propre culture valorisés par un Autre qui représente, à son tour, une culture valorisée - ce qu'on peut admettre pour les États-Unis dans un certain nombre de domaines - conduit naturellement à une auto-évaluation positive.

\section{Conclusion}

59 La présente analyse a permis de mettre en regard deux discours promotionnels de registres contrastés, voire antonymiques: d'une part, un discours promotionnel soigneusement élaboré au cours des siècles pour véhiculer une image d'acceptabilité en termes de "secret, sainteté et santé » qui valorise l'histoire mouvementée de la liqueur de la Chartreuse, le secret de sa fabrication, et la subtilité et le raffinement de ses arômes naturels, et, d'autre part, le succès emporté par le discours promotionnel inopiné issu d'une scène tapageuse qui se situe dans un contexte transgressif de vulgarité, crime, sexe et violence au Texas.

Cependant, une étude portant sur le lien entre un film et ses retombées commerciales sur les ventes du produit qui y figure ne peut que susciter des interrogations quant aux stratégies marketing relevant du " placement du produit». Celui-ci est une stratégie de marketing qui consiste soit à payer pour que le produit soit évoqué ou montré de manière plus ou moins discrète dans une œuvre de fiction, soit à payer un auteur pour intégrer le produit dans le tissu du narratif fictionnel, l'exemple le plus célèbre étant le roman The Bulgari Connection (2001) écrit par Fay Weldon, une auteure à qui le joaillier Bulgari a versé la somme de 18000 livres pour réaliser le projet (Hardy, 2007).

61 D'après Samuel Loutaty, le premier placement de produit au cinéma date de 1916 lorsque « Henry Ford fit la publicité à peine déguisée de son modèle $\mathrm{T}$, tout juste sorti des usines, dans un film muet She Wanted a Ford» (2012, p. 88). Dans un contexte de publicité moins 
flagrant et plus rapproché de notre problématique, Mireille Hardy (2007, p. 188) nous indique que John Huston a été le premier metteur en scène à utiliser le placement de produit au cinéma pour l'alcool, Gordon's gin, dans son film African Queen (1951).

$\mathrm{Vu}$ le rôle que joue la Chartreuse verte dans au moins deux films de Tarantino - Boulevard de la mort (2007) et Inglourious Basterds (2009) - des interrogations quant à une éventuelle stratégie de placement de produit ne peuvent que se poser. La réponse serait négative. L'entreprise, Chartreuse Diffusion, réfute catégoriquement toute suggestion d'une telle démarche commerciale. À cela s'ajoutent les propos du directeur général de l'époque, qui déclare dans une interview publiée en décembre 2011 dans GRENews, un journal gratuit de la région de Grenoble : « Le film de Tarantino a créé un incroyable buzz, et on n'y est pour rien." Le directeur admet même que cela n'a pas été sans engendrer quelques complications pour la société qui n'avait en rien prévu une telle hausse des ventes et a dû mobiliser des employés au service embouteillage en heures supplémentaires pour faire face à la demande.

Une deuxième indication qui tend à confirmer l'absence de placement de produit vient de Tarantino lui-même. Connu pour le rôle important qu'il attribue à des marques, réelles ou imaginaires, dans ses films, il répond à la question dans une interview disponible sur YouTube en disant qu'il a, à ses débuts, effectivement cherché ce type de financement mais, sans doute en raison de sa réputation, a essuyé un refus catégorique. Ce qui expliquerait, peut-être, pourquoi il recourt si souvent à la mise en fiction de marques imaginaires...

\section{BIBLIOGRAPHIE}

ATHERTON Mike, Gambling, Londres, Hodder \& Stoughton, 2006.

BROWN Jared et MILLER Anistatia, Spiritous Journey. A History of Drink, Book One (UK), Mixellany Books, 2001.

FITZGERALD Scott. F., The Great Gatsby, New York, Scribners, 1925.

FREYSSINEY-DOMINJON Jacqueline et WAGNER Anne-Catherine, L'alcool en fête : manière de boire de la nouvelle jeunesse étudiante, Paris, L'Harmattan, 2003.

HARDY Mireille, «Quand l'entreprise s'intéresse à la littérature... », Les Cahiers de l'ILCEA, n 9, L'implicite et les écrits de l'entreprise (dir. Shaeda Isani), 2007, p. 185-202.

ISANI Shaeda, «Le discours des marchands du péché et l'implicite de la quête d'honneur : le

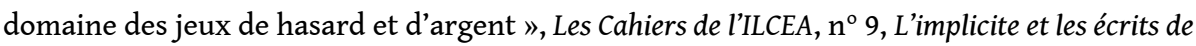
l'entreprise (dir. Shaeda Isani), 2007a, p. 73-97.

-, « Discourse and Counter Discourse in the "Sin" Industries: The Case of the Gambling Industry in the United States", ASp, la revue du GERAS, ${ }^{\text {os }}$ 51/52, 2007b, p. 115-131. 
-, « Euphemisation and the Discourse of Positive Self-Representation: Corporate Image Laundering in the Vice Industries ", dans Denis Jamet et Manuel Jobert (dir.), Empreintes de l'euphémisme. Tours et détours, Paris, L'Harmattan, 2010, p. 107-118.

LOUTATY Samuel, « La saga des publicités masquées », M, le magazine du Monde, 2012, p. 87-89. MASLOW Abraham, Motivation and Personality, New York, Harper, 1954.

MUNRO H. H., Collected Short Stories of Saki [1904], Herefordshire, Wordsworth Classics, 1993.

STEINMETZ Michel, Chartreuse, Grenoble, Glénat, 2006.

WAUGH Evelyn, Brideshead Revisted: The Sacred and Profane Memoirs of Captain Charles Ryder [1945],

Londres, Chapman and Hall, 1964, p. 63.

WELDON Fay, The Bulgari Connection, Londres, Atlantic Monthly Press, 2001.

\section{Références Internet}

«Chartreuse verte » (consulté le 28 janvier 2014).

Cinémiam, « La sorcière verte » (consulté le 28 janvier 2014).

«Elixir végétal - Publicités anciennes » (consulté le 28 janvier 2014).

GRENews (2011), « La liqueur des moines chartreux à l'assaut des comptoirs américains » (consulté le 28 janvier 2014).

L'Artisanat Monastique (consulté le 28 janvier 2014).

« La fabrication aujourd'hui - Chartreuse » (consulté le 28 janvier 2014).

« Les Français boivent trop » (consulté le 24 janvier 2014).

Nielsen-CGA, « Le mojito en tête dans les cafés-hôtels-restaurants français » (consulté 19 février 2014).

OFDT, «Quantité d'alcool consommé par habitant âgé de 15 ans et plus depuis 1961 » (consulté le 28 janvier 2014).

Score3.fr, Renseignements financiers sur les entreprises (consulté le 28 janvier 2014).

« Tarantino talks about product placement in his movies », novembre 2011 (consulté le 28 janvier 2014).

\section{NOTES}

1. Il s'agit d'un alcool élaboré par les moines chartreux en 1605 sous forme d'un élixir créé à partir de plantes. En 1764 apparaît le produit phare de la gamme, la Chartreuse verte, $55^{\circ}$, qui représente aujourd'hui $70 \%$ de la production, et en 1838 la Chartreuse jaune qui titre $40^{\circ}$ et représente $10 \%$ de la production. Jusqu'à nos jours, le secret de la fabrication de la liqueur est gardé par les moines.

2. Notre traduction: "Les gens peuvent dire ce qu'ils veulent au sujet de la décadence du christianisme; mais le système religieux qui a produit la Chartreuse verte ne peut jamais vraiment mourir. »

3. Notre traduction: «La vraie Chartreuse v-v-verte, faite avant l'expulsion des moines. Il y a cinq parfums distincts qui se succèdent sur la langue. C'est comme si on dégustait un arc en ciel de couleurs. » 
4. Quelques-uns des prix et honneurs reçus par Tarantino : 1994 Palme d'or pour Pulp Fiction; 2007 Président du jury du Festival de Cannes; 2011 César d'honneur pour l'ensemble de sa carrière ; 2013 Oscar pour le meilleur scénario original (Django Unchained).

5. Death Proof n'est pas le seul film où la Chartreuse verte entre dans l'imaginaire cinématographique du réalisateur. Dans Inglourious Basterds (2009), la Chartreuse fait une nouvelle mais brève apparition dans une scène clé qui se déroule dans un bar, réunissant, comme aime le faire Tarantino, un Anglais, un Allemand, un Américain et un Français.

6. Traduction : « La seule liqueur si bonne qu'elle a donné son nom à une couleur. »

7. L'éloge fait par Tarantino de la Chartreuse verte dans Boulevard de la mort renvoie à deux domaines référentiels en anglais, la liqueur et la couleur. La mise en évidence de ce deuxième domaine a également eu des retombées inattendues. En effet, en s'inspirant de la couleur très particulière de la Chartreuse verte, le terme chartreuse ou chartreuse green - prononcée " chartrouse »-, pour désigner une nouvelle teinte entre le jaune et le vert, a été adopté en anglais en 1987 pour désigner l'une des couleurs tertiaires de la roue HSV élaborée pour faciliter l'usage des couleurs sur le net, aussi connue comme les 12 couleurs du web. L'existence de cette nouvelle couleur est restée largement inconnue du grand public jusqu'à ce que Tarantino l'évoque. Depuis lors, chartreuse est non seulement une couleur bien connue des anglophones - déclarée couleur de l'année 2011 - mais est également une couleur très à la mode dans de multiples domaines de consommation comme les vêtements, la décoration intérieure, les arts déco, l'art, et même l'horticulture où il existe désormais une rose baptisée "Chartreuse rose ». L'équivalent en français serait peut-être la couleur " anis », qui, elle, fait référence à une autre boisson bien française.

8. Cette entreprise s'est avérée délicate dans la mesure où Google.com englobe tous les pays anglophones et nous ne sommes pas parvenus à cibler la recherche exclusivement vers les ÉtatsUnis. Cependant, bien que les résultats obtenus incluent également des pays comme le RoyaumeUni, l'Australie, le Canada, etc., en partant du prémisse que les États-Unis sont considérés comme le berceau de la mixologie (l'art de l'élaboration des cocktails) et que le terme « cocktail » y a été publié pour la première fois le 28 avril 1803 d'après Jared Brown et Anistatia Miller (2009), historiens des spiritueux et fondateurs du Musée américain des cocktails, nous pouvons formuler l'hypothèse que les résultats obtenus convergent vers les autres indicateurs pour confirmer les tendances américaines dans ce domaine.

9. Initialement, nous avions retenu l'entrée [nom + hot cocktail] mais avons dû l'abandonner, car les résultats furent parasités par l'interprétation littérale de « hot » (boissons chaudes).

10. Le "Green chaud» semble être devenu la boisson identitaire des sports d'hiver, de nombreuses stations de ski proposant cette boisson gracieusement aux visiteurs lors du traditionnel «pot d'accueil».

11. Les résultats obtenus pour l'entrée [nom + cocktail tendance] ont été globalement du même ordre.

12. Nous avons également tenté une recherche relative à une autre boisson alcoolisée semblable, l'Irish Coffee ou café irlandais. La recherche n'a pas pu être concluante, car « café irlandais » est peu utilisé en France et l'entrée «Irish Coffee » s'est évidemment étendue au-delà de la sphère francophone. 


\section{RÉSUMÉS}

La présente étude analyse le traditionnel discours promotionnel d'acceptabilité orchestré autour de la liqueur de la Chartreuse selon les termes du triptyque discursif de «secret, sainteté et santé ", et son évolution suite aux restrictions imposées par le législateur et à son appropriation par le cinéma américain. Nous nous intéressons particulièrement aux retombées commerciales et sociétales induites par la mise en fiction de la Chartreuse verte dans le film Boulevard de la mort de Quentin Tarantino (2007). Ce discours promotionnel sérendipiteux mais puissant, à contrecourant de l'image véhiculée par un discours promotionnel centenaire, en changeant les perceptions des consommateurs américains, a modifié également celles de la jeunesse française, transformant ainsi une liqueur traditionnellement prisée en tant que digestif en cocktail tendance de la jeunesse française.

This study analyses the traditional promotional discourse of acceptability regarding Chartreuse liqueurs orchestrated in terms of a discursive triptych of "secrecy, spirituality and health" and its subsequent evolution, firstly due to restrictions imposed by the legislator and then by its appropriation by Quentin Tarantino in Death Proof (2007). We analyse the commercial and societal impact of the film's serendipitous but nevertheless powerful promotion of Green Chartreuse on the perceptions of American consumers and, by ricochet, those of young consumers in France, thus transforming a liqueur traditionally valued for its digestive powers into a trendy cocktail for the young.

INDEX

Mots-clés : Chartreuse verte, Tarantino, Boulevard de la mort, discours promotionnel, cocktails, jeunesse française

Keywords : Green Chartreuse, Tarantino, Death Proof, promotional discourse, cocktails, French youth

\section{AUTEUR}

\section{SHAEDA ISANI}

Université Grenoble Alpes (France), ILCEA 